\title{
Éditorial - Chantiers pour une décennie nouvelle
}

Editorial - Into a new decade: setting priorities in ESP research

\section{Anthony Saber}

\section{OpenEdition}

Journals

Édition électronique

URL : https://journals.openedition.org/asp/6196

DOI : 10.4000/asp.6196

ISSN : 2108-6354

\section{Éditeur}

Groupe d'étude et de recherche en anglais de spécialité

\section{Édition imprimée}

Date de publication : 1 mars 2020

Pagination : 1-4

ISSN : 1246-8185

\section{Référence électronique}

Anthony Saber, «Éditorial - Chantiers pour une décennie nouvelle », ASp [En ligne], 77 | 2020, mis en ligne le 01 mars 2020, consulté le 21 mars 2022. URL : http://journals.openedition.org/asp/6196 DOl : https://doi.org/10.4000/asp.6196

Ce document a été généré automatiquement le 21 mars 2022.

Tous droits réservés 


\title{
Éditorial - Chantiers pour une décennie nouvelle
}

\author{
Editorial - Into a new decade: setting priorities in ESP research
}

\author{
Anthony Saber
}

1 Le présent numéro contient deux articles de recherche. Catherine Resche explore certains néologismes récemment apparus dans le secteur anglophone de la banque et de la finance. Souvent fondés sur des matrices lexicales telles que l'oxymore, l'amalgame ou les mots-valises, ils reflètent l'émergence de positionnements éthiques nouveaux chez les acteurs du domaine, que l'on a pu traiter de «banksters" dans le sillage de la grande crise financière de 2008. Comme dans de nombreux autres secteurs du monde de l'entreprise, les nouvelles préoccupations liées à la responsabilité sociale, citoyenne et environnementale favorisent l'apparition d'une terminologie méliorative, fondée sur des syntagmes nominaux comprenant certains mots-slogans (responsible, sustainable, impact, mission), comme dans mission-driven finance ou ethical funds. L'emblématique slogan de la finance de marché - «Greed is good» - immortalisé par le banquier d'affaires Gordon Gekko dans le film Wall Street d'oliver Stone ${ }^{1}$ se trouve ainsi démenti en apparence, mais on peut s'interroger, avec C. Resche, sur la compatibilité de ces attitudes responsables avec l'hubris inhérent aux marchés financiers dérégulés.

2 Margaux Guillerit présente un travail intéressant sur les syntagmes nominaux complexes (SNC) en génie civil, mené en collaboration avec l'équipe du Civil Engineering Writing Project (CEWP), coordonné par Susan Conrad (Portland State University). De manière quelque peu contre-intuitive, l'écrit est en effet un enjeu très important dans cette discipline, qui implique la rédaction fréquente de mémorandums et de rapports techniques. En outre, les entreprises du secteur signalent que les jeunes diplômés des instituts de génie civil ne disposent pas des compétences rédactionnelles attendues lorsqu'ils commencent leur carrière, ce qui souligne un enjeu de formation linguistique spécialisée dans ce secteur économique. Exploitant des corpus étiquetés comprenant différents types de rapports techniques, M. Guillerit compare la typologie, la qualité et la densité des SNC dans les productions écrites d'étudiants et de professionnels. Comme Douglas Biber et Bethany Gray $(2010,2011)$ l'ont démontré de manière très 
convaincante, les SNC sont en effet le principal indicateur de la "complexité " syntaxique des styles scientifiques et techniques, où il s'agit souvent d'exprimer de manière concise et claire des concepts sophistiqués. De manière assez surprenante, $\mathrm{M}$. Guillerit constate que le niveau de complexité syntaxique est à peu près le même chez les jeunes diplômés et les acteurs confirmés, même si la fréquence de certains indicateurs diffère (l'étude montre aussi, constat qui était plus prévisible, une forte influence du genre de discours sur la fréquence et la nature de ces structures dans les deux populations). Ce résultat inattendu appelle donc des travaux ultérieurs, pour mieux comprendre par exemple la corrélation entre l'usage des SNC et le contenu thématique de chaque phrase.

3 Ces deux travaux illustrent certains des chantiers qui pourraient être, pour l'anglais de spécialité, ceux des années à venir : veille terminologique avec $\mathrm{C}$. Resche, investigations sur les discours de domaines spécialisés encore peu couverts par nos travaux de recherche, comme le génie civil avec M. Guillerit. Puisque nous nous situons à l'orée d'une décennie nouvelle, il ne nous semble pas interdit de formuler certaines hypothèses, ou même certains souhaits quant au tour que pourrait prendre la recherche en anglais de spécialité.

Une meilleure couverture de la palette des discours spécialisés semble ainsi nécessaire. Les discours des sciences humaines et sociales, des disciplines artistiques demeurent très largement un angle mort de la recherche en anglais de spécialité. Comme l'a très bien montré Jean-Louis Trouillon (2009) pour les discours de l'histoire, la spécialisation de l'anglais y est sans doute plus discrète, moins spectaculaire que dans les discours juridiques, scientifiques et techniques, mais ses formes différentes nous obligent aussi à une réflexion épistémologique renforcée sur la nature même de la spécialisation langagière.

5 Les chantiers à ouvrir semblent non moins innombrables et cruciaux en matière de didactique au sein du secteur LANSAD. Renforcement de la réflexion théorique sur les concepts, développement de modélisations des actions de formation et des interactions entre les acteurs, mise en place d'expérimentations de terrain de type evidence-based (à l'image d'Esther Duflo, récent prix Nobel d'économie, qui a transposé avec succès à la sphère micro-économique les méthodes issues des essais cliniques randomisés en médecine ${ }^{2}$ ), pour tester et valider de nouvelles formules pédagogiques, réexamen ou approfondissement des procédures d'évaluation et de certification (nous rappelons à nos lecteurs que nous souhaitons consacrer le prochain numéro de la revue aux certifications linguistiques), meilleure codification des besoins linguistiques propres à chaque sphère spécialisée...

6 Un troisième type de chantier a trait aux cadres théoriques et aux instruments méthodologiques de l'anglais de spécialité qui, comme l'on sait, s'appuie souvent sur d'autres disciplines de la connaissance pour mener à bien ses investigations. Les travaux de D. Biber et B. Gray cités plus haut illustrent ainsi les liens étroits et anciens entre anglais de spécialité et linguistique, et démontrent la possibilité (du moins pour étudier certains discours ou certains faits de langue) d'une véritable linguistique $d u$ spécialisé, qui pourrait progressivement prendre son autonomie en tant que branche à part entière des sciences du langage. Plus généralement, les liens entre les différentes écoles linguistiques et l'anglais de spécialité ont probablement vocation à être mieux formalisés qu'aujourd'hui, car l'hétérogénéité des positionnements théoriques et des cadres d'analyse nuit sans doute à l'émergence de cette «linguistique du spécialisé » 
que nous appelons de nos vœux. Il nous semble également que l'anglais de spécialité, déjà familier des cadres d'analyse que propose la linguistique pour les discours écrits (les travaux sur la syntaxe sont ainsi fréquents, la linguistique de corpus permet de défricher de vastes corpus spécialisés et de comprendre la phraséologie et les phénomènes collocationnels qui s'y déploient), devrait développer ses liens avec ces autres branches de la linguistique que sont la phonétique-phonologie et la pragmatique, afin de promouvoir une meilleure connaissance de l'oral anglophone spécialisé. Des travaux prometteurs (mais encore trop rares) explorent déjà cette veine de recherche, notamment chez les chercheurs anglophones : on lira par exemple avec intérêt l'étude menée par Julia Trippe et Melissa Baese-Berk (2019) sur la prosodie de l'anglais de l'aviation américain, ou encore celle de Noel Kwan et Katie Dunworth (2016) sur les stratégies pragmatiques dans les interactions entre personnel domestique et employeurs à Hong Kong.

7 Un quatrième chantier réside très probablement dans l'extension des travaux de "terrain» en anglais de spécialité, tant on ressent le besoin de modéliser la spécialisation de l'anglais au plus près des pratiques concrètes des acteurs. Si Dominique Maingueneau signalait dès 1992 le « tour ethnolinguistique » pris par l'École française d'analyse du discours, on constate avec Séverine Wozniak (2019) ${ }^{3}$ que l'anglais de spécialité est lui aussi appelé à cette mutation méthodologique ${ }^{4}$, déjà amorcée dans la décennie 2000 par certains travaux marquants, tels que celui de Louhiala-Salminen en 2002 sur les pratiques discursives routinières d'un cadre dans une entreprise d'informatique finlandaise.

8 Enfin, il est impossible de négliger l'irruption de l'intelligence artificielle dans tous les secteurs des sciences et techniques, eu égard aux percées récentes en matière de réseaux neuronaux informatisés et d'algorithmes de deep learning. L'impact le plus immédiatement visible de ces avancées au sein de la communauté des anglicistes de spécialité réside très certainement dans l'automatisation croissante de la traduction spécialisée, qui modifie de manière substantielle les rôles traditionnels du traducteur (sans doute rapidement appelé à devenir exclusivement réviseur de premières traductions réalisées par des machines) et du traductologue (qui devra de plus en plus inclure dans ses réflexions une interface avec les technologies informatiques). Et nous ne parlons pas ici des logiciels de traduction pour smartphones qui, reposant également sur l'intelligence artificielle, commencent déjà à mettre la traduction simultanée de conversations familières à la portée de tous, et ce dans des dizaines de langues. Évolution technologique de rupture qui, si l'on se livre à un effort de prospective, s'étendra sans doute rapidement aux interactions verbales plus spécialisées, et remettra profondément en cause les rôles classiques de l'enseignant de langues au sein $\mathrm{du}$ secteur LANSAD. Évolution qui mérite donc, très certainement, une réflexion approfondie dès à présent, afin que le professeur comme le chercheur ne soient pas les spectateurs impuissants de ces bouleversements, mais en deviennent des acteurs de premier plan...

9 Ce très bref panorama - non exclusif, très certainement - des pistes qui se dessinent aujourd'hui pour la recherche en anglais de spécialité, illustre le type de travaux que nous souhaiterions pouvoir publier dans la revue ASp au cours de la décennie qui s'ouvre.

10 Vous trouverez également dans le présent numéro trois comptes rendus d'expérience pédagogique par Nadežda Stojković, Alice Henderson et Richard Cauldwell, et 
Evegueniya Lyu, ainsi que trois recensions, par John Humbley, Fanny Domenec et Dacia Dresden-Hammouda.

\section{BIBLIOGRAPHIE}

BIBER, Douglas \& Bethany GRAY. 2010. « Challenging stereotypes about academic writing: Complexity, elaboration, explicitness ». Journal of English for Academic Purposes 9/1, 2-20. BIBER, Douglas \& Bethany GRAY. 2011. « Grammatical change in the noun phrase: The influence of written language use ». English Language and Linguistics 15/2, 223-250.

BOESKY, Ivan. 1985. Merger Mania-Arbitrage: Wall Street's Best Kept Money-Making Secret. New York : Holt Rinehart \& Winston.

KWAN Noël \& Katie DUNWORTH. 2016. «English as a lingua franca communication between domestic helpers and employers in Hong Kong: A study of pragmatic strategies ». English for Specific Purposes 43, 13-24.

ISANI, Shaeda. 2014. «Ethnography as a research-support discipline in ESP teaching, learning and research in the French academic context ». ASp 66, 27-39.

LOUHIALA-SALMINEN, Leena. 2002. «The fly's perspective: discourse in the daily routine of a business manager ». English for Specific Purposes 21/3, 211-231.

MAINGUENEAU, Dominique. 1992. « Le tour ethnolinguistique de l'analyse du discours ». Langages $105,114-125$.

TRIPPE, Julia \& Melissa BAESE-BERK. 2019. « A prosodic profile of American Aviation English ».

English for Specific Purposes 53, 30-46.

TROUILLON, Jean-Louis. 2009. «Ébauche d'une caractérisation de l'anglais de l'histoire ». ASp 56, 527.

WOZNIAK Séverine. 2019. Approche ethnographique des langues spécialisées professionnelles. Berne : Peter Lang, Collection « Aspects linguistiques et cultures des discours spécialisées », dirigée par Catherine Resche.

\section{NOTES}

1. Le célèbre discours de Gordon Gekko (interprété par Michael Douglas) sur la cupidité dans le film d'Oliver Stone avait été inspiré par un personnage réel, Ivan Boesky, banquier d'affaires influent à Wall Street dans les années 1970-1980. Auteur d'un ouvrage sur l'arbitrage d'actifs (Boesky 1985), professeur vacataire de finance à Columbia et à la New York University, il prononça en mai 1986 un discours lors de la cérémonie de remise des diplômes à la School of Business Administration de l'Université de Berkeley. Cette intervention contenait ce passage : "Greed is all right, by the way. I want you to know that. I think greed is healthy. You can be greedy and still feel good about yourself." Voir à ce sujet cet article: <https:// 
www.eleconomista.es/business-insider/noticias/4147412/07/12/Meet-Ivan-Boesky-TheInfamous-Wall-Streeter-Who-Inspired-Gordon-Gecko.html>.

2. Voir à ce sujet <https://www.lequotidiendumedecin.fr/actus-medicales/sante-publique/prixnobel-deconomie-une-francaise-une-approche-originale-basee-sur-la-methodologie-des-essais> et, pour un regard plus critique <https://www.cairn.info/revue-philosophia-scientiae-2019-2page-85.html>.

3. Voir la recension de cet ouvrage dans ce numéro.

4. On lira avec profit à ce sujet l'article de Shaeda Isani (2014), qui préconise un rapprochement entre anglais de spécialité et approches ethnographiques.

\section{AUTEUR}

\section{ANTHONY SABER}

Rédacteur en chef. anthony.saber@ens-paris-saclay.fr 